\title{
Integral definida na geometria: tarefas para o cálculo de volumes
}

\section{André Luis Trevisan}

Docente, Universidade Tecnológica Federal do Paraná, UTFPR, campus Londrina. andrelt@utfpr.edu.br

\section{Higgor Henrique Dias Goes}

Graduando, Universidade Tecnológica Federal do Paraná, UTFPR, campus Londrina. higgorhenrique05@gmail.com

\section{Resumo}

A proposta de tarefas aqui apresentada explora uma das aplicações do cálculo integral de uma variável: o volume de sólidos gerados a partir da revolução de uma curva em relação a um eixo. Além do auxílio de recursos tecnológicos para a visualização, a tarefa intenta relacionar tópicos da geometria espacial como ponto de partida para a obtenção de uma fórmula geral do volume de um sólido gerado pela revolução de curvas quaisquer.

Palavras-chave: Educação Matemática. Ensino de Cálculo Diferencial e Integral. Tarefas matemáticas. Recursos tecnológicos.

\section{Definite integral in geometry: tasks for calculating the volume}

\begin{abstract}
This tasks runs one of the applications of a variable integral calculation: volume solids generated from the revolution of a curve about an axis. Besides the aid of Technological resources for the visualization, the task tries to relate the spatial geometry topics as a starting point for obtaining a formula of the volume of the solid generated by the revolution of any bends.

Keywords: Mathematics Education. Teaching Differential and Integral Calculus. Mathematical tasks. Technological resources.
\end{abstract}

\section{Introdução}

Nos processos de ensino e aprendizagem da Matemática, os estudantes precisam ter um papel ativo trabalhando, sempre que possível, em grupos e em tarefas não precedidas pela apresentação de uma definição ou exemplo similar. O papel do professor é incentivá-los a apresentar e discutir suas 
ideias durante as realizações das tarefas, e conduzir a sistematização dos conceitos subjacentes. Assim, antes de introduzir um conceito mediante sua definição formal, o estudante é convidado a explorá-lo intuitivamente. Tais elementos constituem o que estamos denominando ambientes de ensino e aprendizagem pautados em episódios de resolução de tarefas. Ao pensar o desenho das tarefas, destacamos a importância da incorporação de recursos tecnológicos. Concordamos com Borba, Silva e Gadanidis (2015, p. 48) quando propõem a noção de experimentação com tecnologias como "o uso de tecnologias informáticas no estudo de conceitos ou na exploração de problemas matemáticos".

Neste contexto, apresentamos uma proposta para aulas de Cálculo Diferencial e Integral 1 (CDI-1), inspirada nas ideias de Freudenthal (1973, 1991). Para ele, o ensino dessa disciplina deveria ser precedido pela exploração qualitativa, intuitiva e informal de ideias como taxa de variação e áreas sob curvas, por meio de abordagens gráficas e numéricas, gradativamente refinadas. Volumes e áreas, densidades, velocidades e outros conceitos físicos e cinemáticos podem ser calculados de forma intuitiva sem que definições mais gerais tenham sido apresentadas.

\section{Proposta de tarefa}

São propostas duas tarefas (Figuras 1 e 2) que têm como público-alvo estudantes de CDI-1 já "apresentados" ao conceito de integral definida de uma função potência por meio, por exemplo, do método da exaustão (TREVISAN; GOES, 2016).

Sua organização por meio de questões do tipo "aberto controladas" leva os estudantes a transporem conceitos aprendidos em um contexto (área do segmento parabólico) para outro (volume de sólidos de revolução). A mediação pelas potencialidades de recursos tecnológicos faz com que assuma um caráter de descoberta/reinvenção (FREUDENTHAL, 1973, 1991).

\section{Figura 1 - Tarefa 1}

Um sólido de revolução é gerado pela rotação de uma região plana em torno de uma reta no mesmo plano da região. A reta é denominada eixo de rotação.

1. Em cada caso, esboce o sólido obtido pela rotação, em torno do eixo $x$, da região delimitada pela função dada no intervalo $[0, b]$.
i) $\quad f(x)=x^{0}$
ii) $f(x)=x$
iii) $f(x)=x^{2}$
iv) $f(x)=\sqrt{x}$

2. Utilizando conhecimentos de Geometria Espacial, determine em termo de $b$, fórmulas para o volume dos dois primeiros sólidos. Levante alguma hipótese sobre o volume dos demais.

Fonte: Elaborada pelos autores. 
Figura 2 - Tarefa 2

Execute o aplicativo [figura abaixo]. Movimente o seletor $\mathbf{n}$ e observe o que acontece nas duas telas. Seja $f(x)=x^{2}$ e o intervalo $[1,3]$.

a) O que está sendo representado nesse aplicativo? Descreva.

b) Adote $n=4$. Represente, na forma de Soma de Riemman, a aproximação do volume do sólido. Calcule essa soma.

c) É possível melhorar a estimativa obtida no item anterior.

i) Explique como.

ii) Apresente uma integral resultante desse processo.

iii) Utilize sua "descoberta" para avaliar a hipótese levantada na parte 1 da tarefa.

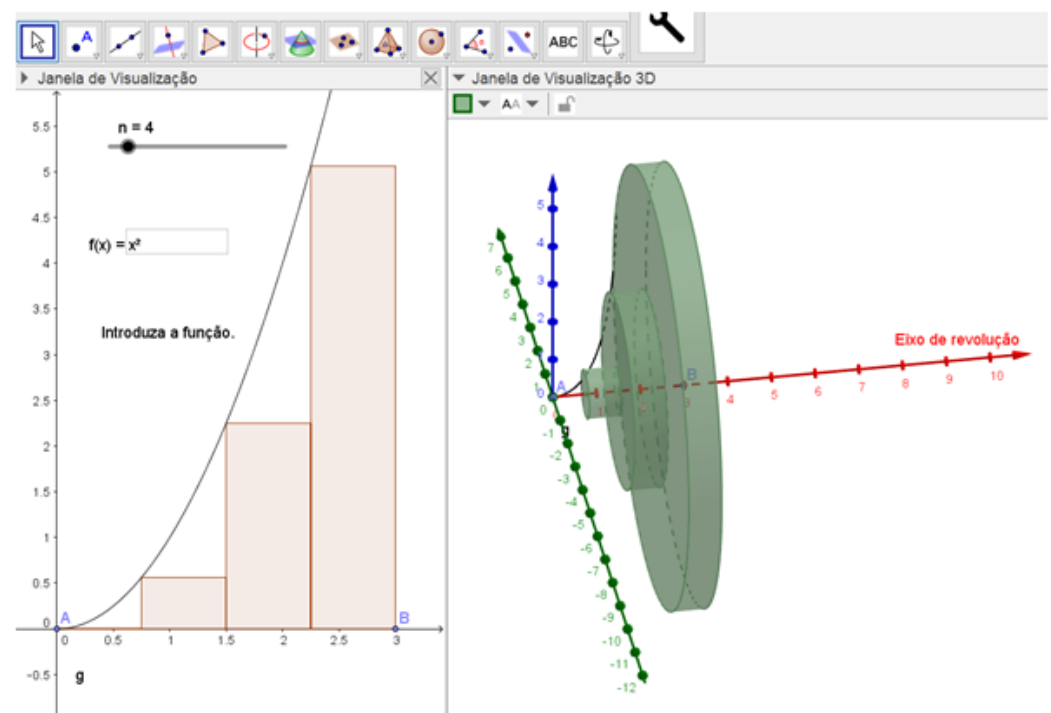

Fonte: Elaborada pelos autores.

\section{Discussão}

Na Tarefa 1, para calcular o volume dos sólidos dos itens (i) e (ii), os estudantes podem utilizar fórmulas usualmente vistas no Ensino Médio: na primeira situação um cilindro e na segunda um cone (Figura 3), ambos retos e com altura $b$. O raio da base do cilindro será 1 e do cone será a ordenada do ponto de abscissa $b$ (no caso, $b$ ).

Figura 3 - Volume dos sólidos.

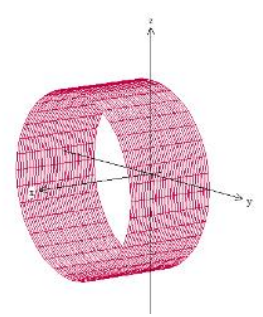

cilindro

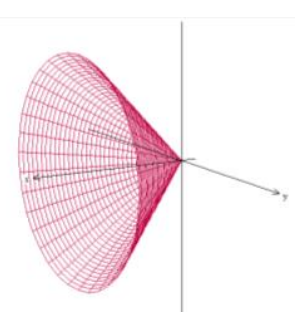

cone

Fonte: Elaborada pelos autores.

São então instigados a buscar algum padrão nas fórmulas dos volumes desses dois sólidos: ambos calculados pelo produto da área da base e altura, sendo no cone multiplicado por $\frac{1}{3}$. Então, para $f(x)=x^{0}$ o volume é $\pi b$ e para $f(x)=x^{1}$, é $\pi \frac{b^{3}}{3}$. Do esboço dos sólidos dos itens (iii) e (iv) (Figura 4), observa-se que volume é menor quando aumenta o expoente da função $f(x)=x^{n}$, e 
o padrão sugere uma expressão da forma $\pi \frac{b^{p}}{p}$, com $p$ desconhecido e passível de investigação (por meio da Tarefa 2).

Figura 4 - Sólidos obtidos pela rotação de funções.

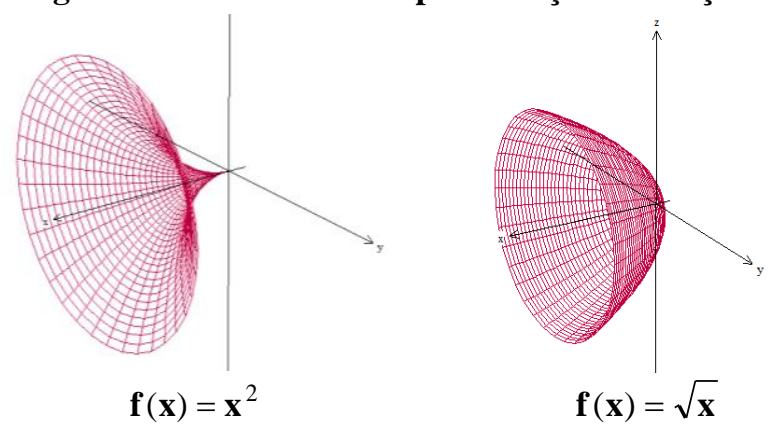

Fonte: Elaborada pelos autores.

Dispondo de arquivo do Geogebra, os estudantes são então convidados a confrontar seus esboços iniciais com uma construção na qual é possível alterar tanto a expressão da função quanto o intervalo em $x$ que delimita a região a ser rotacionada. $\mathrm{O}$ controle deslizante permite visualizar o fatiamento do sólido e contribui na elaboração de um modelo de aproximação do volume utilizando Somas de Riemann. Cada fatia "infinitesimal” é aproximada por um cilindro, com volume igual ao produto da área de sua seção transversal (um círculo de área $\pi[f(x)]^{2}$ ) pela altura "infinitesimal” $\Delta x$. O volume do sólido por ser aproximado por $\sum_{i=1}^{n} \pi[f(x i)]^{2} \Delta x$.

Analisando o que acontece quando tomamos valores de $n$ "muito grandes" (o que pode ser feito de modo intuito, com auxílio de controle deslizante que indica o número de retângulos da subdivisão - sem necessidade de formalização do conceito de limite), inferimos que o volume converge com valor exato $\int_{0}^{b} \pi[f(x)]^{2} d x$.

Por fim, podem confrontar suas hipóteses iniciais com os resultados obtidos a partir desse processo de generalização. No caso particular em que $f(x)=x^{n}, n \in I N$, o volume será $\pi \frac{b^{2 n+1}}{2 n+1}$, resultado de $\pi \int_{0}^{b}\left(x^{n}\right)^{2} d x$. Uma discussão quanto à extensão desse resultado para o caso $n \in \mathfrak{R}$ pode ser fomentada a partir da investigação da validade dessa fórmula para o caso em que $f(x)=\sqrt{x}$.

Como "fechamento", uma discussão sobre as condições de validade da fórmula construída (no caso, para funções quaisquer contínuas no intervalo $[0, b]$ ) e outras investigações considerando novas configurações (um intervalo $[a, b]$; sólidos gerados a partir da rotação de regiões delimitadas por duas curvas; sólidos que tenham "buraco"; rotação em torno de outros eixos) podem ser 
realizadas.

\section{Considerações finais}

Do processo de elaboração das tarefas destacamos alguns elementos que consideramos importantes na organização de um ambiente de aprendizagem para aulas de CDI pautado em episódios de resolução de tarefas: i) ao invés de uma sequência de "exercícios" de aplicação de uma fórmula previamente "exposta", propõe-se aqui um trabalho com situações que, gradativamente, possibilitam a "reinvenção" de conceitos; ii) a atitude do professor, enquanto "instigador" por meio de questionamentos torna possível pensar um ambiente de aprendizagem na qual se desenvolvem competências para a resolução de problemas; iii) há possibilidade de os estudantes organizarem matematicamente fenômenos, dos quais a formalização é uma consequência do trabalho gradativo (e não uma condição inicial necessária).

Lançamos mão de recursos tecnológicos que, associados à proposição de tarefas do tipo “aberto controladas", assumem um papel fundamental na elaboração e no desenvolvimento de nossa proposta didática, oferecendo "caminhos propícios para processos como a formulação de conjecturas, realização de testes, refinamento de conjecturas, familiarização com notações" (BORBA; SILVA; GADANIDIS, 2015, p. 55).

\section{Agradecimentos}

Agradecemos à Fundação Araucária e ao CNPq (Processo 457765/2014-3).

\section{Referências}

BORBA. M.C.; SILVA, R.S.R; GADANIDIS, G. Fases das tecnologias digitais em Educação Matemática, Sala de aula e internet em movimento. Belo Horizonte: Autêntica, 2015. (Coleção Tendências em Educação Matemática).

FREUDENTHAL, H. Mathematics as an educational task. Dordrecht: D. Reidel Publishing Company, 1973.

. Revisiting Mathematics Education. Netherlands: Kluwer Academic Publishers, 1991.

TREVISAN, A. L.; GOES, H. H. D. O método da exaustão e o cálculo de áreas: proposta de uma tarefa com auxílio do GeoGebra. Educação Matemática em Revista (São Paulo), v. 52, p. 79-85, 2016. 